\title{
The Effect of Spacer Length on the Polymerization of Diacetylenes in SAMs on Gold Surfaces
}

\author{
Henning Menzel ${ }^{1}$, Mark D. Mowery ${ }^{2}$, Mei Cai ${ }^{2}$, Christine E. Evans ${ }^{2}$ \\ ${ }^{1}$ Universität Hannover, Institut für Makromolekulare Chemie, \\ Am Kleinen Felde 30, 30167 Hannover, Germany \\ 2 University of Michigan, Chemistry Department, \\ 930 N. University Ave., Ann Arbor, MI 48109-1055, USA
}

\begin{abstract}
SUMMARY: The influence of the molecular architecture of diacetylene disulfides on the structure and polymerizability of self assembled monolayers on gold was investigated. The position of the diacetylene group was varied systematically by adjusting the length of the tail and the spacer. For the strutures studied, the longer the tail, the higher the akyl chain crystallinity as sensed by IR spectroscopy, and the higher the polymerization efficiency as probed by resonance Raman spectroscopy. The influence of the spacer on the polymerizability is more complicated. It is conjectured that the spacer reduces the lattice strain due to the misfit of the arrangement of the anchor groups and the arrangement of the diacetylene units and the polydiacetylene backbone, respectively. Simple modeling reveals that the number of the methylene units (odd or even) and the overall length both influence the arrangement. The highest polymerization efficiency is found for the longest, odd numbered spacer.
\end{abstract}

\section{Introduction}

Poly(diacetylene)s (PDA) exhibit an electronic transition due to the extented $\pi$-system. Characterized extensively as crystals and LB films, ${ }^{1-14)}$ polydiacetylenes are known to exist in several phases which appear blue, purple, or red in color. Although the exact nature of this chromism remains elusive, it is generally attributed to variations in the effective conjugation length, which is determined by the length of the polymer chain, the chain conformation and the local environment of the polymer backbone. ${ }^{11)}$ Because the excitonic absorption is directly related to this effective conjugation length, the blue phase is characterized by the longest conjugation length with the purple and the red forms indicative of successively diminished conjugation length. ${ }^{3-7,15,16}$ Furthermore, both reversible and irrreversible phase transitions, particularly between the blue and red phases, have been observed. PDAs in LB films ${ }^{15,17,18)}$ or in liposomes ${ }^{19,20}$ ) have been suggested for application in colorimetric sensors. In these sensor designs, the conjugation length of the polymer is conjectured to be diminished upon interaction of the target molecule with the diacetylene bilayer, leading to a color change response. For such sensor applications it is therefore of great importance to obtain polymer with a long effective conjugation length, i.e. blue phase polymer. However, the constraints for the topochemical polymerization of diacetylenes are considerable ${ }^{12)}$ and poly(diacetylene)s with a long conjugation length (blue phase polymer) can be obtained only if the monomeric assembly is in a highly ordered state which meets these constraints (see Figure 1). 


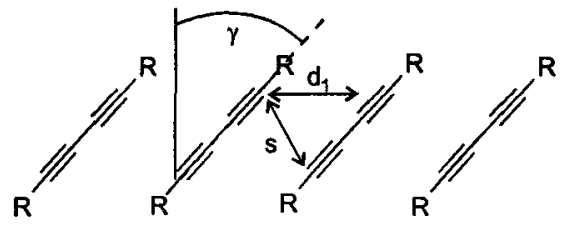

$$
\lambda<350 \mathrm{~nm}\left\lfloor\begin{array}{l}
d_{1}=d_{2}=4.7-5.2 A \\
s=3.4-4.0 \AA \Rightarrow \gamma \approx 45^{\circ}
\end{array}\right.
$$

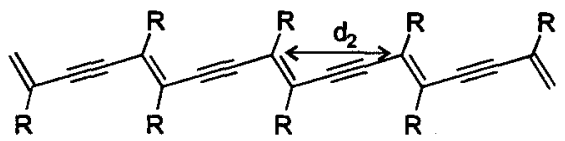

Figure 1: $\quad$ Steric requirements for the polymerization of diacetylenes.

For LB films incorporating diacetylene moieties, the interdependence between the molecular architecture and the structure and polymerizability is well studied, ${ }^{3,5-9,11)}$ Recent studies have shown that poly(diacetylene) assemblies also can be prepared as self-assembled monolayers (SAMs). ${ }^{21-25)}$ These SAMs are of particular interest because they are much more stable than LB films due to the covalent binding to the substrate. ${ }^{26)}$ Furthermore, the preparation of SAMs is not limited to planar substrates. ${ }^{27)}$ However, in self-assembled monolayers, the interaction between the headgroup and the surface is much stronger than in LB films due to the covalent linkages at the surface. As a result interdependence between molecular structure and polymerizability observed for LB films may not be directly applicable for predicting the properties of these surface attached diacetylene monolayers. In this report the influence of changes in the molecular architecture on the structure of the SAMs and their impact on the monolayer structure and the polymerization process are examined using various photopolymerizable diacetylenic disulfides:

$$
\begin{aligned}
& \mathrm{CH}_{3}-\left(\mathrm{CH}_{2}\right)_{n}-\mathrm{C} \equiv \mathrm{C}-\mathrm{C} \equiv \mathrm{C}-\left(\mathrm{CH}_{2}\right)_{\mathrm{m}}-\mathrm{S} \\
& \mathrm{CH}_{3}-\left(\mathrm{CH}_{2}\right)_{n}-\mathrm{C} \equiv \mathrm{C}-\mathrm{C} \equiv \mathrm{C}-\left(\mathrm{CH}_{2}\right)_{\mathrm{m}}-\mathrm{S}
\end{aligned}
$$

The architecture of the monomolecular assemblies was adjusted synthetically by varying both the tail $(n=7,11,15)$ and spacer $(m=4,6,9)$ length. $\left.{ }^{28}\right)$ A $n, m-D A$ and $n, m-P D A$ designation is used to identify the nonpolymerized and the polymerized monolayer architectures, respectively. The overall film quality was investigated employing contact angle measurements. ${ }^{25}$ ) The internal order of the monolayers was probed by use of grazing angle infrared spectroscopy ${ }^{25,29)}$ and the polymerization was monitored by resonance Raman spectroscopy. 25,30 )

\section{Results}

\section{Wettability}

To investigate the interdependence of the molecular architecture and the polymerizability of diacetylene containing SAMs, several diacetylene containing disulfides were synthesized ${ }^{28)}$ and 
self-assembled from solution onto gold surfaces. ${ }^{29)}$ The overall quality of these films was investigated by contact angle measurements. Although a large lateral area is probed when a drop of solvent is placed on the monolayer, interactions probe only the outermost methylene region. As shown in Tab. 1, contact angles for water on the longest tail monolayers $(15,9$ PDA; 15,6 PDA; 15,4 PDA) are almost identical and in good agreement with monolayers formed from octadecane thiol (C18). In contrast, contact angles exhibit a significant diminuition as the length of the tail region is decreased (11,9 PDA, 7,9 PDA). ${ }^{25)}$ This result is likely due to an increase of the disorder in the short tail chain, exposing more methylene groups to the surface as has been observed for short alkanethiols. ${ }^{31)}$ The contact angle measurements indicate a high overall film quality, where the tail region influences the outer chain ordering.

Tab. 1: Advancing contact angles for water on polymerized self-assembled monolayers of diacetylenic disulfides on gold ( \pm standard deviation of five measurements).

\begin{tabular}{lcccccc}
\hline & 15,4 PDA & 15,6 PDA & 15,9 PDA & 11,9 PDA & 7,9 PDA & C18 \\
\hline contact angle & $110 \pm 3,7$ & $109 \pm 3,9$ & $109 \pm 1,8$ & $105 \pm 3,4$ & $96 \pm 2,0$ & $113 \pm 2,2$ \\
\hline
\end{tabular}

\section{Polymerization}

UV/Vis spectroscopy is the method of choice to monitor the polymerization of diacetylene compounds since the conjugated polymer backbone shows a strong absorption in the visible region. However, this method is not applicable to SAMs on gold due to the overlap of the polymer absorption band with the plasmon bands of the substrate. ${ }^{25}$ ) Resonance Raman spectroscopy provides a good alternative approach due to the selective measurement of the conjugated polymer backbone. Indeed the resonant enhancement of the Raman signal for the polymer backbone allows monitoring of the polymerization process without interference from nonresonant transitions. Alkyl-based monolayers and nonpolymerized diacetylenes do not show any signal under the conditions used in this study. Furthermore, photoselection in resonant Raman spectroscopy can be used to discriminate the blue and red polymer phases. Excitation at $633 \mathrm{~nm}$ is expected to be in resonance with the blue phase polymer only, while excitation at $532 \mathrm{~nm}$ probes both blue and red phase polymer. ${ }^{7,25,30,32)}$

The spectra obtained for the SAMs at two excitation wavelengths $(633 \mathrm{~nm}$ probing blue phase polymer and $532 \mathrm{~nm}$ probing both blue and red phase polymer) are shown in Figure 2. The transitions are assigned to vibrations in the double $\left(1450-1500 \mathrm{~cm}^{-1}\right)$ and triple $(2080-2100$ $\mathrm{cm}^{-1}$ ) bond regions. As expected for a highly conjugated system, these transitions are considerably shifted relative to the transitions anticipated for the isolated bonds (1620 and $2260 \mathrm{~cm}^{-1}$, respectively. ${ }^{33)}$ The transition in the $700 \mathrm{~cm}^{-1}$ region has not been conclusively assigned in the literature and is conjectured to arise from the in-plane stretching vibrations of the backbone. ${ }^{7,21)}$ Relative intensities of alkene and alkyne vibrational transitions at a given excitation wavelength are expected to correspond with the degree of polymerization into the 
specific form. However, intensity comparison between spectra obtained with differing excitation wavelengths is not feasible due to different experimental configurations.
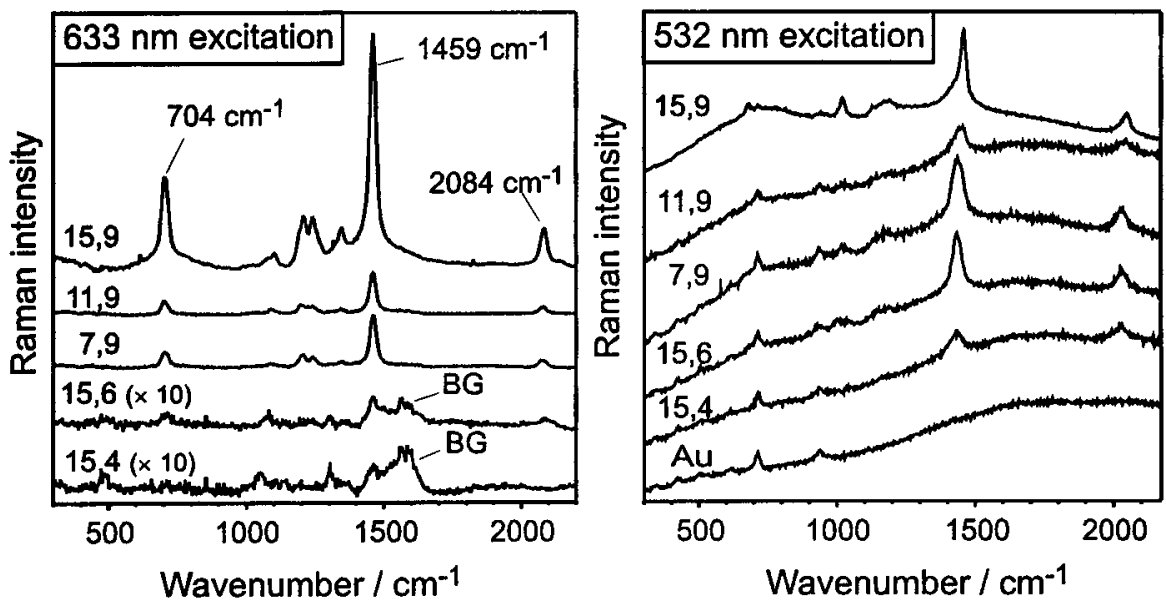

Figure 2: Resonance Raman spectra for two excitation wavelengths as function of molecular architecture (different scaling, see text).

The spectra indicate that for all molecular architectures polymerization can be observed, however the polymerization efficiency is clearly different. For 15,9 PDA there is a significant concentration of blue phase polymer, but for 11,9 PDA and 7,9 PDA lower intensities in the spectra for $633 \mathrm{~nm}$ excitation indicate less blue phase polymer. As the spacer length is decreased $(15,9$ PDA $\rightarrow 15,6$ PDA $\rightarrow 15,4$ PDA) the blue form decreases markedly until no signal is observed for the shortest spacer. However, excitation at $532 \mathrm{~nm}$, which probes both red and blue phase polymer, shows the presence of polymer for all molecular architectures. For the shortest spacer the signal is small, indicating that the 15,4 PDA has only a small degree of polymerization. ${ }^{25}$ )

\section{Alkyl chain crystallinity}

In LB film studies, decreasing alkyl chain crystallinity has been correlated with decreasing conjugation length, leading to the red-phase polymer. This correlation may arise from a decrease in the polymer length caused by a misalignment of neighboring diacetylenes with more disordered alkyl chains. Equally likely, disordered alkyl chains place strain on the polymer backbone, decreasing the conjugation length. The alkyl chain crystallinity can be probed by infrared spectroscopy. A highly crystalline, all-trans configuration is expected to exhibit the asymmetric $\left(v_{\mathrm{a}}-\mathrm{CH}_{2}\right)$ and symmetric $\left(\mathrm{v}_{\mathrm{s}}-\mathrm{CH}_{2}\right)$ methylene stretching transitions at $2919 \mathrm{~cm}^{-1}$ and $2850 \mathrm{~cm}^{-}$ 1, respectively. ${ }^{34)}$ The transition frequency for both transitions will be shifted to higher wavelengths for decreasing order. As can be seen in Figure 3, the spectra of the monolayers vary considerably. The IR spectra indicate some disorder for the shorter tail structures $(11,9$ 
DA and 7,9 DA), while the longest tail structures of $15,9,15,6$ and 15,4 show peak positions indicating a highly ordered structure (see Tab. 2).

Tab. 2: Band positions for the methylene stretch vibrations in monomeric and polymeric diacetylene SAMs

\begin{tabular}{ccccc}
\hline & \multicolumn{2}{c}{$\mathrm{v}_{\mathrm{a}}-\mathrm{CH}_{2}$} & \multicolumn{2}{c}{$\mathrm{v}_{\mathrm{s}}-\mathrm{CH}_{2}$} \\
& DA & PDA & DA & PDA \\
\hline 15,4 & 2919 & 2919 & 2851 & 2851 \\
15,6 & 2919 & 2919 & 2851 & 2851 \\
15,9 & 2919 & 2919 & 2852 & 2851 \\
11,9 & 2924 & 2924 & 2855 & 2855 \\
7,9 & 2925 & 2926 & 2855 & 2855 \\
octadecane thiol & \multicolumn{3}{c}{2920} & \multicolumn{3}{c}{2851} \\
\hline
\end{tabular}

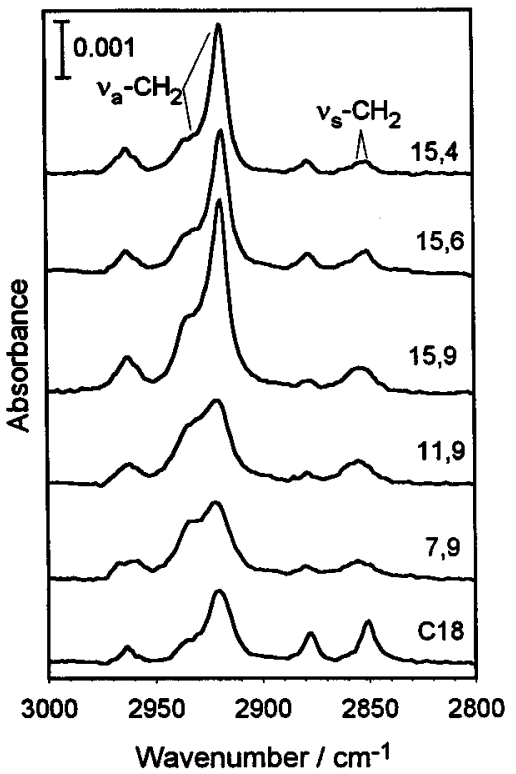

Figure 3: Fourier transform infrared spectra of the $\mathrm{CH}$ stretching region for diacetylene SAMs with various molecular architectures obtained at grazing incidence

However, upon increasing the spacer length from four to nine methylene units, an increasing transition appears as a higher frequency shoulder on the $\mathrm{v}_{\mathrm{a}}-\mathrm{CH}_{2}$ and $\mathrm{v}_{\mathrm{s}}-\mathrm{CH}_{2}$ peaks. The position of the transition is consistent with a non-ordered polymethylene chain. This transition is ascribed to an increased disordered component within the alkyl chains. The nonhomogeneity may arise from a domain structure with disordered chains at the domain boundaries. However, the high intensity of the disordered component in the IR spectra especially for the $15,9 \mathrm{DA}$ monolayer is not consistent with electrochemical experiments, which indicate a very low defect density due to domain boundaries. ${ }^{25,29)}$ Alternatively, a model where the spacer and the tail have different methylene chain structures has been proposed. ${ }^{29)}$ In this model a tightly packed 
tail region provides a methyl surface with high blocking capability, masking a more loosely packed spacer region. It is important to note that a decreased crystallinity, as sensed by the IRmeasurements for the spacer region, is not associated solely with the absence of order. It would also be observed if the spacer is in an arrangement with reduced chain-chain interactions, a so-called "conformationally ordered liquid state".

\section{Discussion}

Monitoring the polymerization of diacetylene SAMs with two excitation wavelengths revealed that the polymerization efficiency clearly depends on the molecular architecture of the compounds self- assembled onto the gold surface. High concentrations of blue phase polymer are found for the 15,9 PDA. Upon decreasing the length of the alkyl tail i.e. for 11,9 PDA and 7,9 PDA, the concentration of blue phase polymer is reduced significantly. Both the wettability and IR studies, which indicate more disorder in the SAMs with decreasing tail length, are consistent with disorder as origin of the limited polymerization efficiency. This observation is in accordance with LB film studies, which have shown that a high degree of order is necessary in the side chain region to obtain good polymerization efficiency. ${ }^{8,11)}$ However, decreasing order cannot explain the significantly less efficient polymerization as the spacer length is decreased. Monolayers of 15,6 PDA and 15,4 PDA exhibit only little or no signal, when blue phase polymer is probed, although wettability and IR spectra indicate highly ordered alkyl chains in both cases. Indeed, the disordered portion, which manifests itself as a high frequency shoulder, is decreasing when the spacer becomes shorter. Since polymerizability is best for a long spacer, which shows a significant disordered portion, it can be conjectured that the spacer acts primarily to accomodate the alignment of adjacent diacetylene moieties. The longer spacer chain offers increased degrees of freedom and reduces the lattice strain from the misfit between the arrangement of the anchor groups at the gold surface and the packing of the diacetylene moieties. However in this case odd and even spacers are also expected to result in slightly different arrangements of the diacetylene units. For simple alkyl thiols it has been demonstrated that end group orientation is depending on the number of the methylene units. ${ }^{35}$ Although a two-dimensional representation is always inadequate to give a clear picture of the SAM structure, the structure of diacetylene containing alkyl chains on gold as depicted in Figure 4 readily shows differences for odd and even numbered spacers. The structures have been drawn according to the knowledge about the structure of alkyl thiols on gold. ${ }^{31,36)}$ The distance of the sulfur atoms is $4.99 \AA^{31)}$ The tilt angle of the alkyl chains $\theta$ depicted here, is slightly smaller than the value determined for pure alkylthiols $\left.\left(\theta \sim 30^{\circ}-40^{\circ}\right) .31,34\right)$ However, the tilt of the alkyl chain is a convolution of the tilt in the CCC-plane (paper plane) and perpendicular to the CCC-plane. There is experimental evidence that in the case of the diacetylene containing monolayers the tilt perpendicular to the CCC-plane is more important than for pure alkyl thiols ${ }^{29}$ ) and therefore a smaller tilt in the CCC-plane, as drawn here, is reasonable. Furthermore, there is experimental evidence that the diacetylene moieties form an internal scaffolding. ${ }^{29,37)}$ The arrangement of the diacetylene moieties therefore is assumed to be very 
similiar to that found in crystals (i.e. $\gamma \sim 45^{\circ}$ ). The major difference between the structures for even and odd numbered spacers, respectively, is the difference in the angle $\alpha$ (gold-sulfur-C). This angle should be $109^{\circ}$ for a $\mathrm{sp}^{3}$-hybridizised sulfur. ${ }^{36)}$ While $\alpha$ is close to $109^{\circ}$ for even numbered spacers, odd numbered all-trans spacers would result in an $\alpha$ of almost $180^{\circ}$. Therefore, some gauche bonds have to be in the odd numbered spacer chains and a higher level of disorder may be expected.

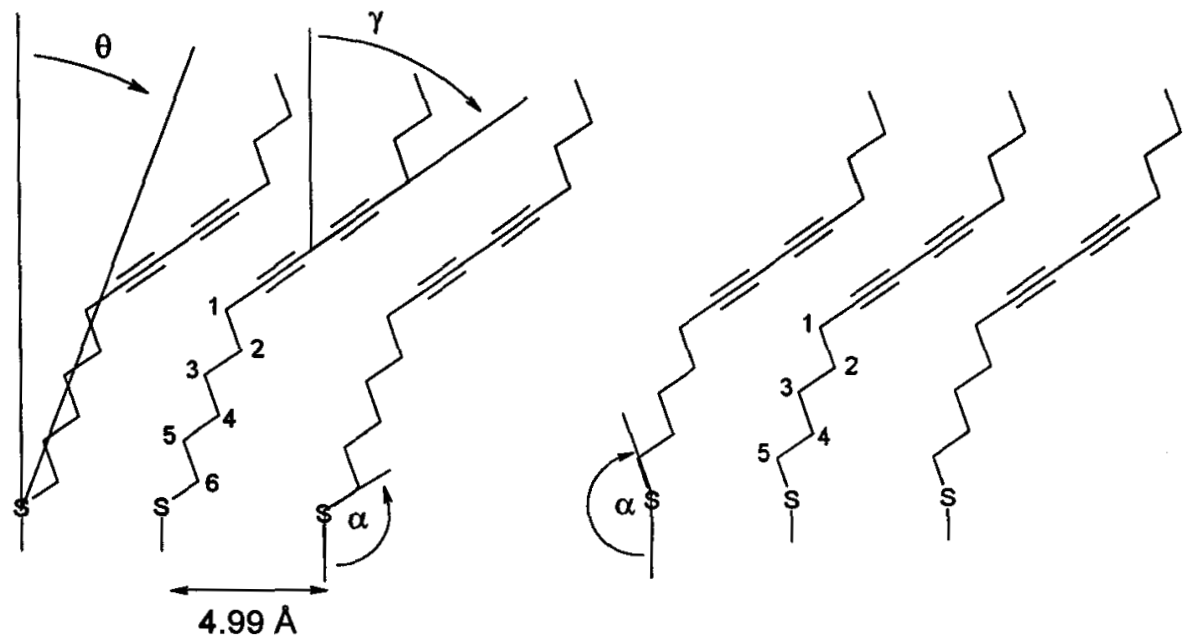

Figure 4: Schematic representation of the arrangement in a diacetylene containing monolayer for an even ( 6 methylene units, left) and odd (five methylene units, right) numbered spacer.
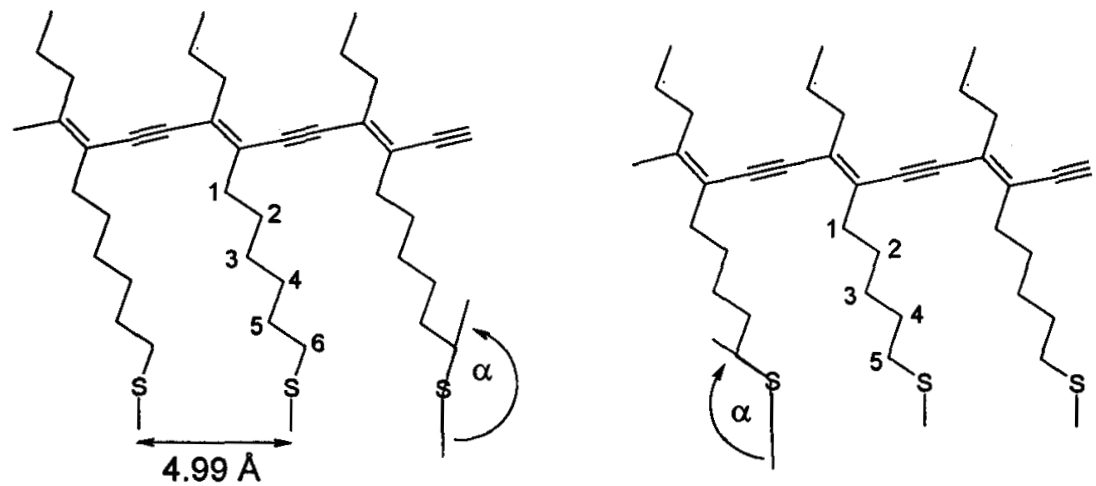

Figure 5: Schematic representation of the arrangement in a polydiacetylene monolayer for an even ( 6 methylene units, left) and odd (five methylene units, right) numbered spacer.

Although a packing favorable for the polymerization can be adapted in the case of even numbered spacers without gauche bonds, this does not necessarily imply that the 
polymerization is more favorable than in the case of odd numbered spacers. Because of the hybridization change in the diacetylene moiety upon polymerization, some structural changes have to take place within the spacer region. Figure 5 depicts a two-dimensional representation of the structure in a polymerized SAM. Again, the major difference between the structures for even and odd numbered spacers, respectively, is the difference in the angle $\alpha$ (gold-sulfur-C). However, upon polymerization and the concomitant change of the hybridization, an odd numbered spacer becomes more favorable. In polydiacetylene SAMs $\alpha$ is close to $109^{\circ}$ for odd numbered spacers and would be almost $180^{\circ}$ for even numbered spacers. This might cause too much strain to obtain the highly conjugated blue phase polymer in the case of even numbered spacers, since the anchor group cannot change its orientation as is possible in LB films. ${ }^{3}$ ) In particular too much strain can be anticipated for spacers too short to accomodate both reasonable bond angle for the anchor group and the change in the hybrization at the polymer backbone, even with gauche conformations. Thus, a decreased polymerization efficiency for diacetylene SAMs with short even numbered spacers can be expected. The increased disordered portion in the diacetylene SAMs with an odd numbered (and longer) spacer as well as the decreased polymerization efficiency for even numbered spacers, are in fact experimentally observed.

\section{Conclusion}

The influence of the molecular architecture of diacetylene containing disulfides on the structure and polymerizability of self-assembled monolayers on gold was investigated. The position of the diacetylene group was varied systematically by adjusting the length of the tail and spacer. The length of the tail group determines the order within the system. The longer the tail the higher the crystallinity of the SAM and the more efficient the polymerization. The spacer mainly acts to accomodate an arrangement of the diacetylene units favorable for polymerization. It can be conjectured that the arrangment is influenced by the length of the spacer and the number of the methylene units (odd or even), since one end of the spacer is tethered to the surface. An even numbered spacer can accomodate an arrangement of the diacetylene units without the need to distort the all-trans configuration, while some gauche bonds are neccessary for an odd numbered spacer. However, upon polymerization and the concomitant change of the hybridization, an odd numbered spacer is more favorable. So the molecular architecture of the diacetylene compound not only has to allow an arrangement of the triple bonds favorable for polymerization, but also to accomodate both the change in hybrization and a reasonable bond angle at the anchor group. To substantiate the conclusion drawn here, additional architectures have to be investigated and a more sophisticated modeling of the monolayer structure has to be applied to the system.

\section{References}

1) G. Wegner, Z. Naturforsch. B 24, 824 (1969)

2) B. Tieke, G. Wegner, D. Naegle, H. Ringsdorf, Angew. Chem. Int. Ed. Engl. 15, 764 (1976) 
3) B. Tieke, H.J. Graf, G. Wegner, B. Naegele, H. Ringsdorf, A. Bauerjie, D. Day, J.B. Lando, Colloid Polym. Sci. 255, 521 (1977)

4) G. Lieser, B. Tieke, G. Wegner, Thin Solid Films 68, 77 (1980)

5) D. Day, J.B. Lando, Macromolecules 13, 1478 (1980)

6) D. Day, J.B. Lando, Macromolecules 13, 1483 (1980)

7) B. Tieke, D. Bloor, R.J. Young, J. Mater. Sci. (London) 17, 1156 (1982)

8) B. Tieke, G. Lieser, J. Colloid Interface Sci. 88, 471 (1982)

9) B. Tieke, G. Lieser, K. Weiss, Thin Solid Films 99, 95 (1983)

10) V. Enkelmann, Adv. Polym. Sci. 63, 91 (1984)

11) B. Tieke, Adv. Polym. Sci. 71, 79 (1985)

12) J.B. Lando, in: "Polydiacetylenes" D. Bloor, R.R. Chance Eds., Nijhoff, Dordrecht, The Netherlands (1985), pp. 363

13) M. Schott, G. Wegner, in: "Nonlinear optical properties of organic molecules and crystals", Eds. D.S. Chemla, J. Zyss, Academic Press, Orlando (1987)

14) G. Cao, T.E. Mallouk, J. Solid State Chem. 94, 59 (1991)

15) N. Mino, H. Tamura, K. Ogawa, Langmuir 7, 2336 (1991)

16) K. Kuriyama, H. Kikuchi, T. Kajiyama, Langmuir 12, 2283 (1996)

17) D.H. Charych, J.O. Nagy, W. Spevak, M.D. Bedvarski, Science 261, 585 (1993)

18) Q. Cheng, R. Stevens, Adv. Mater. 9, 481 (1997)

19) A. Reichert, J.O. Nagy, W. Spevak, D. Charych, J. Am. Chem. Soc. 117, 829 (1995)

20) J.J. Pan, D. Charych, Langmuir 13, 1365 (1997)

21) D.N. Batchelder, S.D. Evans, T,L. Freeman, L. Häussling, H. Ringsdorf, H. Wolf, $J$. Am. Chem. Soc. 116, 1050 (1994)

22) T. Kim, Q. Ye, L. Sun, K.C. Chan, R.M. Crooks, Langmuir 12, 6065 (1996)

23) K.C. Chan, T. Kim, J.K. Schoer, R.M. Crooks, J. Am. Chem. Soc. 117, 5875 (1995)

24) M.D. Mowery, C.E. Evans, J. Phys. Chem. B 101, 8513 (1997)

25) H. Menzel, M.D. Mowery, M. Cai, C.E. Evans, J. Phys. Chem. B 102, 9550 (1998)

26) T. Kim, K.C. Chan, R.M. Crooks, J. Am. Chem. Soc. 119, 189 (1997)

27) H. Menzel, M.D. Mowery, M. Cai, C.E. Evans, Adv. Mater. in press (1999)

28) M.D. Mowery, C.E. Evans, Tetrahedron Lett. 38, 11 (1997)

29) H. Menzel, M.D. Mowery, M. Cai, C.E. Evans, Macromolecules submitted

30) M. Cai, M.D. Mowery, H. Menzel, C.E. Evans, Langmuir submitted

31) A. Ulman, "An Introduction to ultrathin Organic Films - From Langmuir-Blodgett to Self-Assembly" Academic Press, San Diego (1991)

32) A.J. Campbell, C.K.L. Davies, D.N. Batchelder, Macromol. Chem. Phys. 199, 109 (1998)

33) D. Lin-Vien, N.B. Colthup, W.G. Fateley, J.G. Grasselli in: "The Handbook of Infrared and Raman Characteristic Frequencies of Organic Molecules", Academic Press, San Diego (1991)

34) M.D. Porter, T.B. Bright, D.L. Allara, C.E.D. Chidsey, J. Am. Chem. Soc. 109, 3559 (1987)

35) P.E. Laibinis, G.M. Whitesides, D.L. Allara, Yu-Tai Tao, A.N. Parikh, R.G. Nuzzo, J. Am. Chem. Soc. 113, 7152 (1991)

36) H. Sellers, A. Ulman, Y. Shnidman, J.E. Eilers, J. Am. Chem. Soc. 115, 9389 (1993)

37) M.D. Mowery, H. Menzel, Mei Cai, C.E. Evans, Langmuir 14, 5594 (1998) 